\title{
Clinical improvement of renal amyloidosis in a patient with systemic-onset juvenile idiopathic arthritis who received tocilizumab treatment: a case report and literature review
}

\author{
Songkiat Chantarogh ${ }^{1 *}$, Soamarat Vilaiyuk ${ }^{2}$, Thipwimol Tim-Aroon ${ }^{3}$ and Suchin Worawichawong ${ }^{4}$
}

\begin{abstract}
Background: Juvenile idiopathic arthritis (JIA) is a common rheumatic disease in children and adolescents. Although JIA may cause secondary amyloidosis, this is a rare complication in patients with JIA and other rheumatic diseases. Many previous studies have revealed that common heterozygous or homozygous mutations in the MEFV gene are associated with systemic-onset JIA (SJIA).

Case presentation: We herein report a case involving a 19-year-old female patient with difficult-to-control SJIA. She developed progressive proteinuria without clinical signs or symptoms of edema. Renal amyloidosis was diagnosed by renal pathologic examination, which demonstrated deposition of eosinophilic amorphous material in the interlobular arteries, arterioles, and interstitium. Electron microscopy showed fibrillary material deposits with a diameter of 8 to $10 \mathrm{~nm}$. A heterozygous E148Q mutation in the MEFV gene was identified. Conventional disease-modifying anti-rheumatic drugs and etanercept had been used to treat the SJIA, but the disease could not be controlled. Therefore, we decided to start tocilizumab to control the disease activity. However, the patient was unable to receive a standard dose of tocilizumab in the early period of treatment because of socioeconomic limitations. Her disease course was still active, and proteinuria was found. Therefore, tocilizumab was increased to a dose of $8 \mathrm{mg} / \mathrm{kg}$ every 2 weeks (standard dose of SJIA), and the patient exhibited a clinical response within 3 months.
\end{abstract}

Conclusion: Refractory SJIA associated with renal amyloidosis is an uncommon cause of proteinuria in adolescents. Tocilizumab may be a beneficial treatment for renal amyloidosis in patients with SJIA.

Keywords: Renal amyloidosis, Systemic-onset juvenile idiopathic arthritis, Tocilizumab

\section{Background}

Amyloidosis is a group of systemic or localized diseases characterized by extracellular deposition of amyloid fibrils in multiple organs $[1,2]$. Organ involvement depends on the subtype of amyloidosis. Secondary or reactive amyloidosis can develop in patients with chronic inflammatory diseases and affects multiple organs such as the kidneys, liver, heart, gastrointestinal system, and autonomic nervous

\footnotetext{
* Correspondence: chantarogh@hotmail.co.th

${ }^{1}$ Division of Nephrology, Faculty of Medicine Ramathibodi Hospital, Mahidol University, Postal address: 270, Rama 6 Road, Phayathai, Ratchathewi,

Bangkok 10400, Thailand

Full list of author information is available at the end of the article
}

system [3]. The amyloidogenic precursor protein in secondary amyloidosis is mainly synthesized by the hepatic system in a chronic inflammatory state. Accumulation of amyloid fibrils leads to progressive dysfunction of affected organs [4]. Juvenile idiopathic arthritis (JIA) is a common rheumatic disease in children and may cause secondary amyloidosis [5, 6]. However, amyloidosis is still a rare complication in patients with JIA and other rheumatic diseases $[5,6]$. Many previous studies have revealed that common heterozygous or homozygous mutations in the $M E F V$ gene are associated with systemic-onset JIA (SJIA) [7-9]. We herein report a case involving an adolescent female patient 
who was diagnosed with SJIA and developed progressive proteinuria. She was found to carry a heterozygous E148Q mutation in the $M E F V$ gene. Renal pathological examination confirmed the diagnosis of amyloidosis. This case will help general practitioners and pediatricians to recognize the etiology of proteinuria in adolescents with JIA.

\section{Case presentation}

\section{Clinical history and investigation results}

A 19-year-old female Thai patient had been diagnosed with SJIA 10 years previously and had been developing progressive proteinuria for 1 year. Her initial manifestations of SJIA were quotidian fever, polyarthritis, and pericardial effusion. Although she received pulse methylprednisolone, high-dose prednisolone, and multiple disease-modifying anti-rheumatic drugs from her previous hospital (including methotrexate, sulfasalazine, and cyclosporine A), her disease activity remained mostly active. At the age of 13 years, she was referred to Ramathibodi Hospital for proper management by a pediatric rheumatologist. Her blood samples were negative for antinuclear antibody, anti-doublestranded DNA, and HLA-B27 with normal levels of C3 $(1550 \mu \mathrm{g} / \mathrm{mL}$; reference range, $900-1800 \mu \mathrm{g} / \mathrm{mL})$ and $\mathrm{C} 4$ (551 $\mu \mathrm{g} / \mathrm{mL}$; reference range, $100-600 \mu \mathrm{g} / \mathrm{mL}$ ). No underlying disease was found in her family members. During the first year of follow-up at Ramathibodi Hospital, the patient was treated with $25 \mathrm{mg} /$ week of etanercept [an anti-tumor necrosis factor (TNF) agent], $25 \mathrm{mg} /$ week of methotrexate, $2 \mathrm{~g} /$ day of sulfasalazine, and $5 \mathrm{mg} /$ day of prednisolone. After 6 months of treatment with etanercept, she still had severe polyarthritis. Therefore, the etanercept was discontinued, and tocilizumab, a humanized anti-interleukin (IL)6 receptor antibody, was started at that time. However, we could not use a standard dose of tocilizumab for SJIA $(8 \mathrm{mg} / \mathrm{kg}$ every 2 weeks) in the early treatment period because of the patient's socioeconomic situation. Therefore, she received tocilizumab at a dose of $8 \mathrm{mg} / \mathrm{kg}$ every 4 weeks. She partially responded to the tocilizumab; her IL-6 level slightly declined from 1105.0 to $574.2 \mathrm{pg} / \mathrm{mL} 5$ months after starting the treatment. Her disease course still waxed and waned. Her arthritis relapsed while receiving the tocilizumab. Therefore, pulse methylprednisolone at $1 \mathrm{~g} / \mathrm{month}$, leflunomide at $20 \mathrm{mg} /$ dose, and hydroxychloroquine at $200 \mathrm{mg} /$ day were gradually added to the tocilizumab regimen. The rheumatologist noticed her first episode of albuminuria when her urine albumin dipstick result was $2+$. This proteinuria showed evidence of progression at her 1-year follow-up, when her urinary protein-to-creatinine ratio (UPCR) exhibited deterioration from 0.87 to 3.00 (normal ratio, <0.2). A nephrologist was then consulted to diagnose the cause of the progressive proteinuria on the background of refractory SJIA.
On physical examination, her vital signs were normal. She was cachectic (body weight, <3rd percentile) and had a short stature (height, $<10$ th percentile). Her wrists, knees, and ankles were stiff and inflamed. There were no signs or symptoms of edema. Other physical findings were unremarkable. Her urinalysis showed an inactive sediment. Significant proteinuria was confirmed by a $24-\mathrm{h}$ urine collection method, which showed a total urine protein of $1295 \mathrm{mg} /$ day $\left(1792 \mathrm{mg} / 1.73 \mathrm{~m}^{2} /\right.$ day). Blood chemistry analysis showed hypoalbuminemia $(2.68 \mathrm{~g} / \mathrm{dL})$, a normal serum cholesterol level $(152 \mathrm{mg} / \mathrm{dL})$, and a normal serum creatinine level $(0.47 \mathrm{mg} / \mathrm{dL})$. Infectious screenings were negative for hepatitis $\mathrm{B}$, hepatitis $\mathrm{C}$, and human immunodeficiency viral infection. Other diagnostic investigation results are listed in Table 1.

\section{Renal biopsy findings}

The renal tissue contained eight nonsclerotic glomeruli. The glomeruli were unremarkable. The mesangium showed focal expansion without hypercellularity. Depositions of acellular eosinophilic amorphous material were seen in the glomerular hilum, mesangium, arteriolar wall, and interstitium (Fig. 1a, b). The material demonstrated fuchsinophilic staining, and Congo red staining was positive (Fig. 1c, d) with apple green birefringence under polarized microscopy. Mild tubular atrophy and interstitial fibrosis were also seen. An immunofluorescence study was negative for IgG, IgM, IgA, C3, C1q, fibrinogen, kappa, and lambda. Electron microscopy revealed randomly oriented fibrils of 8 to $10 \mathrm{~nm}$ in diameter in the mesangium, interstitium, and arteries (Fig. 2a, b). The glomerular basement membrane was unremarkable, but the podocytes showed partial foot process effacement under electron microscopy. These biopsy findings confirmed renal amyloidosis.

Table 1 Clinical characteristics and laboratory results

\begin{tabular}{lll}
\hline Parameters & $\begin{array}{l}\text { Before amyloidosis } \\
\text { treatment }\end{array}$ & At 1-year follow-up \\
\hline $\begin{array}{l}\text { Clinical characteristics } \\
\text { - Number of joints }\end{array}$ & 19 & 11 \\
$\quad$ involved & & \\
- CHAQ-DI score & 1.37 & 1.25 \\
Immunology & & \\
- IL-6, pg/mL & $624.5(0-7)$ & $528.7(0-7)$ \\
- ESR, mm/h & $72(5-20)$ & $10(5-20)$ \\
- CRP, mg/L & $76.23(<5)$ & $3.40(<5)$
\end{tabular}

Data are presented as the laboratory result with reference range in parentheses unless otherwise indicated

CHAQ-DI Childhood Health Assessment Questionnaire Disability Index, IL-6 interleukin-6, ESR erythrocyte sedimentation rate, CRP C-reactive protein 


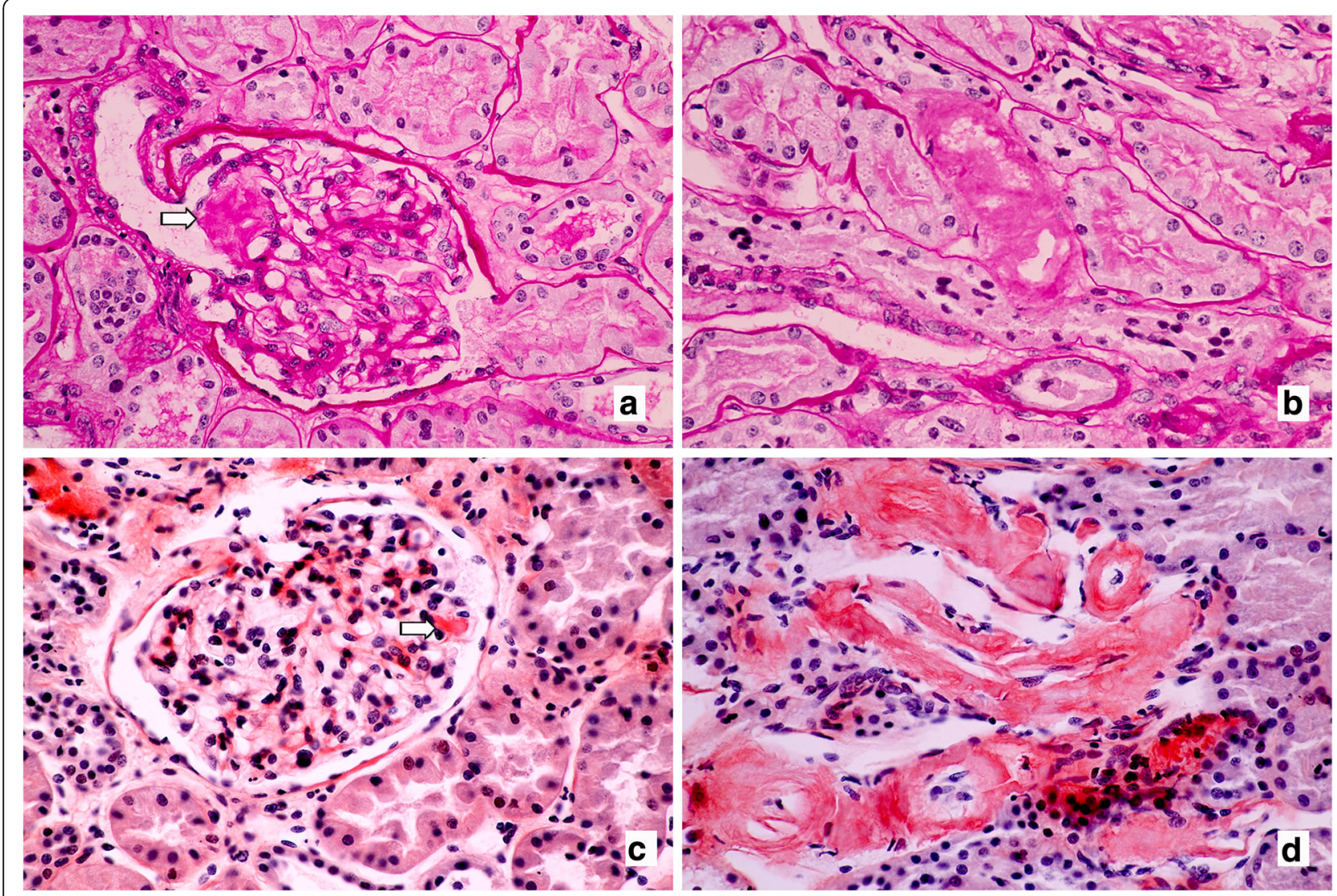

Fig. 1 Depositions of amorphous eosinophilic material (arrow) in the (a) glomerular hilum and (b) arteriolar wall ( $\times 400$, periodic acid-Schiff). Positive Congo red staining in the (c) mesangium and (d) arteriolar wall and interstitium ( $\times 400$, Congo red)

\section{Clinical follow-up after renal biopsy}

Because the amyloidosis was secondary to uncontrolled SIIA, the tocilizumab was increased from a dose of $8 \mathrm{mg} / \mathrm{kg}$ every 4 weeks to a dose of $8 \mathrm{mg} / \mathrm{kg}$ every 2 weeks. We also started enalapril at $5 \mathrm{mg} /$ day $(0.12 \mathrm{mg} / \mathrm{kg} /$ day $)$ for an additional antiproteinuric effect. The other drugs were continued at their same dosages. Two months later, the patient's Childhood Health Assessment Questionnaire Disability Index score decreased. Her UPCR had also decreased from 3.00 to 0.92 , and her arthritis improved
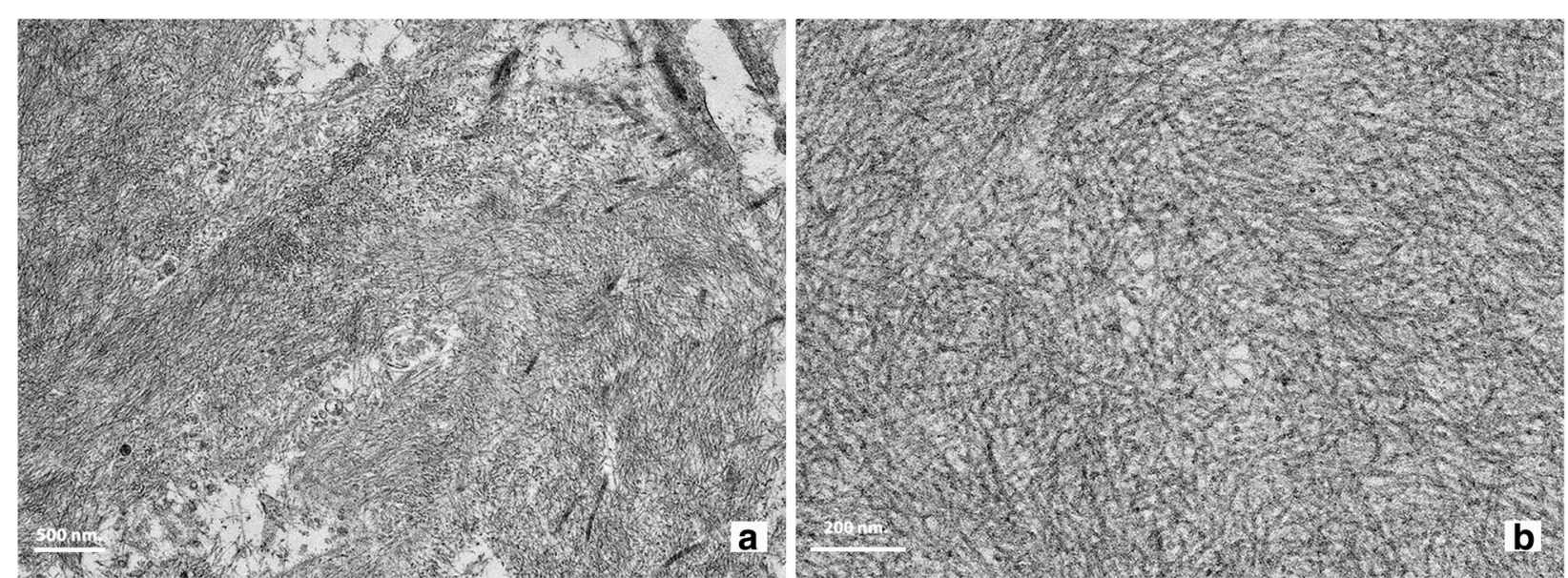

Fig. 2 Electron micrograph shows (a) fibrillary deposits in the arteriolar wall and interstitium $(\times 12,000)$. b Randomly oriented fibrils measuring 8 to $10 \mathrm{~nm}$ in diameter $(\times 40,000)$ 
3 months after the tocilizumab increment. At the 1-year follow-up, her UPCR had decreased to 0.23 and renal function remained stable, with a serum creatinine concentration of around 0.42 to $0.52 \mathrm{mg} / \mathrm{dL}$. Her C-reactive protein concentration had returned to normal and her IL-6 concentration had slightly decreased. Other follow-up investigation results are listed in Table 1.

\section{MEFV mutation analysis}

A 3-mL venous blood sample was collected from the patient for DNA extraction. Next-generation sequencing was performed by SureSelect V5 using the Illumina HiSeq 4000 platform. Sanger sequencing was performed for variant verification. Variants of the $M E F V$ gene (NM\#000243, transcript ID: ENST00000219596) were analyzed. A heterozygous c.442G > C (pE148Q) mutation in the $M E F V$ gene was identified.

\section{Discussion}

Renal amyloidosis is a rare cause of proteinuria in children. The prevalence of amyloidosis is higher in patients with JIA than in the general pediatric population $[10,11]$, and among the seven subtypes of JIA, SJIA is associated with the highest prevalence of amyloidosis $[10,12]$. In the present case, amyloidosis occurred 10 years after the onset of SJIA, similar to a previous study [12]. In many types of amyloidosis, the kidneys are the predominant organ involved. Proteinuria is the most common clinical presentation [5].

The MEFV gene encodes a protein called pyrin or marenostrin, which inhibits the processing of IL- $1 \beta$ to an active form through the regulation of nuclear factorKB and caspase-1 [9]. Pyrin deficiency results in uncontrolled production of active IL-1 $\beta$ [13]. The $M E F V$ gene is also responsible for familial Mediterranean fever, which is an autosomal recessive disorder [13]. Multiple mutations (polymorphisms) of the $M E F V$ gene in one allele have been reported in patients with SJIA [7-9, 14]. The present patient only had a heterozygous E148Q mutation in the $M E F V$ gene, which is a common polymorphism. Whether the E148Q polymorphism is a benign or disease-causing mutation remains controversial. Although glutamic acid has been conserved at position 148 throughout evolution, thus favoring mutation, the general populations of many ethnicities exhibit a high frequency of E148Q (Egyptian, 6.66\%; Jewish, 4.30\%6.60\%; Greek, 1.30\%; Japanese, $23.70 \%$ [9, 14-16]; and Thai, 24.3\% [unpublished data]). Without other polymorphisms in the MEFV gene, the presentation of amyloidosis of our patient may or may not have been related to the heterozygous E148Q mutation. Further functional studies with large samples from both the general population and patients with SJIA are needed to clarify the penetrance of E148Q.
Although we cannot classify the specific subtype of the amyloidogenic precursor in our country, AA amyloidosis is the most common subtype in patients with various chronic inflammatory diseases, including SJIA [3-6]. Therefore, we treated the patient in this case based on current evidence regarding treatment of AA amyloidosis. The main principle of management is suppression of the inflammatory process and control of the underlying disease $[5,17]$. The clinical characteristics and treatment of JIA-related renal amyloidosis are shown in Table 2. Three of six patients were diagnosed with SJIA, and two responded to chlorambucil or leflunomide treatment for renal amyloidosis $[18,19]$. Cantarini et al. [8] could not demonstrate the efficacy of colchicine in one patient with SJIA because of loss to follow-up. Some studies have shown the efficacy of chlorambucil for JIA-related amyloidosis [10, 11]. One case-cohort study [10] revealed that $80 \%$ of patients receiving chlorambucil achieved 10 -year survival compared with only $24 \%$ of patients who had never received cytostatic treatment. However, there is lack of supporting evidence from randomized controlled trials (RCTs) regarding the efficacy of chlorambucil and leflunomide in patients with JIA-related amyloidosis.

Many studies have also demonstrated inadequate efficiency of anti-TNF- $\alpha$ medications for treatment of SJIA; remission was achieved in less than half of all patients in these studies, and relapsing disease was common [20-23]. Two studies have shown the efficacy of anti-TNF therapy in treating amyloidosis secondary to autoimmune diseases, including JIA [24, 25]. A retrospective study of 15 patients with amyloidosis with underlying rheumatic diseases revealed that $54.5 \%$ of patients receiving infliximab (81\%) or etanercept (19\%) showed renal improvement and $17 \%$ showed renal progression at the end of follow-up [25]. Two RCTs demonstrated the efficacy of tocilizumab for the treatment of refractory SJIA in patients without amyloidosis [26, 27]. Several retrospective studies have demonstrated clinical improvement rates of around $70 \%$ in adult patients with rheumatic disease with amyloidosis after tocilizumab treatment $[28,29]$. However, patients with SJIA were not included in these studies; thus, the efficacy of tocilizumab treatment for amyloidosis secondary to SJIA remains unclear.

The present patient developed secondary amyloidosis despite receiving tocilizumab, which can be explained by her uncontrolled SJIA. The patient still had persistent active arthritis while receiving a tocilizumab dose of $8 \mathrm{mg} / \mathrm{kg}$ every 4 weeks. Although she had no systemic features, her inflammatory markers (IL-6 level of $624.5 \mathrm{pg} / \mathrm{mL}$ and C-reactive protein level of $76.2 \mathrm{mg} / \mathrm{L}$ ) were persistently high, reflecting insufficient control of her disease activity. After the tocilizumab was increased from every 4 weeks to 


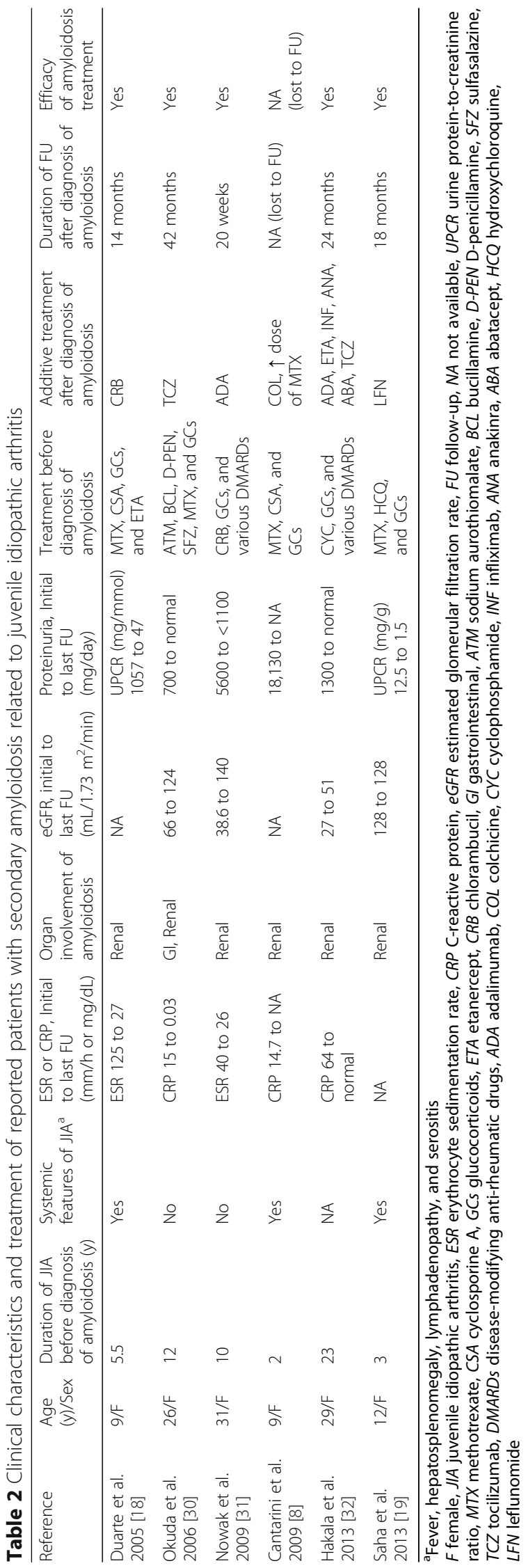


every 2 weeks, which is the same dosage reported in previous RCTs [26, 27], her clinical symptoms of arthritis were relieved and her inflammatory markers decreased within 3 months. She had stable renal function, and her UPCR had decreased to 0.23 at the 1-year follow-up. Therefore, tocilizumab is beneficial in treating amyloidosis secondary to SJIA when conventional therapies fail. However, the minimum dose of tocilizumab in such patients is $8 \mathrm{mg} / \mathrm{kg}$ every 2 weeks.

\section{Conclusion}

We have described a case of renal amyloidosis secondary to refractory SJIA in a carrier of the E148Q mutation in the $M E F V$ gene. Physicians should be aware of the possibility of amyloidosis development in patients with SJIA, especially when such patients show progressive proteinuria and uncontrolled disease. Early detection and appropriate treatment will affect clinical outcomes and lead to a better prognosis. Tocilizumab may be beneficial in patients with amyloidosis related to uncontrolled SJIA.

\begin{abstract}
Abbreviations
ABA: Abatacept; ADA: Adalimumab; ANA: Anakinra; ATM: sodium aurothiomalate; BCL: Bucillamine; CHAQ-DI: Childhood Health Assessment Questionnaire Disability Index; COL: Colchicine; CRB: Chlorambucil; CRP: C-reactive protein; CSA: Cyclosporine A; CYC: Cyclophosphamide; DMARDs: Disease-modifying antirheumatic drugs; D-PEN: D-penicillamine; eGFR: Estimated glomerular filtration rate; ESR: Erythrocyte sedimentation rate; ETA: Etanercept; FU: Follow-up; GCs: Glucocorticoids; Gl: Gastrointestinal tract; HCQ: Hydroxychloroquine; IL: Interleukin; INF: Infliximab; JA: Juvenile idiopathic arthritis; LFN: Leflunomide; MTX: Methotrexate; NA: Not available; RCT: Randomized controlled trial; SFZ: Sulfasalazine; SJIA: Systemic-onset juvenile idiopathic arthritis;

TCZ: Tocilizumab; UPCR: Urine protein-to-creatinine ratio
\end{abstract}

\section{Acknowledgements}

The authors thank the patient and her parent. The authors also thank Dr. Duangrurdee Wattanasirichaigoon and Saisuda Nujarean from the Division of Medical Genetics, Department of Pediatrics, Faculty of Medicine Ramathibodi Hospital, Mahidol University, Thailand for assisting with the genetic analysis.

\section{Funding}

The genetic analysis was funded by the Faculty of Medicine Ramathibodi Hospital, Mahidol University, Thailand. The patient's medical expenses were supported by a grant-in-aid from the Universal Health Care Coverage Project of the National Health Security Office (NHSO), Thailand.

\section{Availability of data and materials}

Variants of the MEFV gene were analyzed and the data of genetic sequencing were cited from NCBI Sequence Read Archive (SRA). The transcript ID was ENST00000219596 and the reference sequence was NM 000243 and NP 000234. The hyperlink for the repository is http://asia.ensembl.org/Homo_sapiens/Gene/ Summary?g=ENSG00000103313;r=16:3242028-3256627.

\section{Authors' contributions}

SC collected the patient's clinical data and was the main contributor to the writing of the manuscript. SV participated in the data collection and reviewed the manuscript. TTA participated in the preparation and discussion of the genetic data. SW participated in preparation of the pathological data. All authors approved the final manuscript.

\section{Competing interests}

The authors declare that they have no competing interests.

\section{Consent for publication}

The patient and her parent received all information regarding this case report. Informed consent for publication was obtained in written form of BMC nephrology from the patient.

\section{Ethics approval and consent to participate}

This case report was approved by the ethic committee of the Faculty of Medicine Ramathibodi Hospital, Mahidol University, Thailand. Protocol number: ID 09-58-37. The patient gave the informed consent for participation in written form of Ramathibodi hospital.

\section{Publisher's Note}

Springer Nature remains neutral with regard to jurisdictional claims in published maps and institutional affiliations.

\section{Author details}

'Division of Nephrology, Faculty of Medicine Ramathibodi Hospital, Mahidol University, Postal address: 270, Rama 6 Road, Phayathai, Ratchathewi, Bangkok 10400, Thailand. 'Division of Rheumatology, Faculty of Medicine Ramathibodi Hospital, Mahidol University, Postal address: 270, Rama 6 Road, Phayathai, Ratchathewi, Bangkok 10400, Thailand. ${ }^{3}$ Division of Medical Genetics, Department of Pediatrics, Faculty of Medicine Ramathibodi Hospital, Mahidol University, Postal address: 270, Rama 6 Road, Phayathai, Ratchathewi, Bangkok 10400, Thailand. ${ }^{4}$ Department of Pathology, Faculty of Medicine Ramathibodi Hospital, Mahidol University, Postal address: 270, Rama 6 Road, Phayathai, Ratchathewi, Bangkok 10400, Thailand.

Received: 27 April 2016 Accepted: 5 May 2017

Published online: 12 May 2017

\section{References}

1. Pettersson T, Konttinen YT. Amyloidosis-recent developments. Semin Arthritis Rheum. 2010;39(5):356-68.

2. Sipe JD, Benson MD, Buxbaum JN, Ikeda S, Merlini G, Saraiva MJ, et al. Amyloid fibril protein nomenclature: 2010 recommendations from the nomenclature committee of the international society of Amyloidosis. Amyloid. 2010;17(3-4):101-4.

3. Bergesio F, Ciciani AM, Santostefano M, Brugnano R, Manganaro M, Palladini $\mathrm{G}$, et al. Renal involvement in systemic amyloidosis-an Italian retrospective study on epidemiological and clinical data at diagnosis. Nephrol Dial Transplant. 2007;22(6):1608-18.

4. Joss N, McLaughlin K, Simpson K, Boulton-Jones JM. Presentation, survival and prognostic markers in AA amyloidosis. QJM. 2000;93(8):535-42.

5. Gillmore JD, Hawkins PN. Pathophysiology and treatment of systemic amyloidosis. Nat Rev Nephrol. 2013;9(10):574-86.

6. Lachmann HJ, Goodman HJ, Gilbertson JA, Gallimore JR, Sabin CA, Gillmore JD, et al. Natural history and outcome in systemic AA amyloidosis. N Engl J Med. 2007:356(23):2361-71.

7. Ayaz NA, Ozen S, Bilginer Y, Erguven M, Taskiran E, Yilmaz E, et al. MEFV mutations in systemic onset juvenile idiopathic arthritis. Rheumatology (Oxford). 2009;48(1):23-5.

8. Cantarini L, Lucherini OM, Simonini G, Galeazzi M, Baldari CT, Cimaz R. Systemic-onset juvenile idiopathic arthritis complicated by early onset amyloidosis in a patient carrying a mutation in the MEFV gene. Rheumatol Int. 2012;32(2):465-7.

9. Lotfy HM, Kandil ME, Issac MS, Salah S, Ismail NA, Abdel Mawla MA. MEFV mutations in Egyptian children with systemic-onset juvenile idiopathic arthritis. Mol Diagn Ther. 2014;18(5):549-57.

10. David J, Vouyiouka O, Ansell BM, Hall A, Woo P. Amyloidosis in juvenile chronic arthritis: a morbidity and mortality study. Clin Exp Rheumatol. 1993; 11(1):85-90.

11. Packham JC, Hall MA. Long-term follow-up of 246 adults with juvenile idiopathic arthritis: functional outcome. Rheumatology (Oxford). 2002;41(12): $1428-35$.

12. Immonen K, Savolainen A, Kautiainen H, Hakala M. Longterm outcome of amyloidosis associated with juvenile idiopathic arthritis. J Rheumatol. 2008; 35(5):907-12.

13. Onen F. Familial Mediterranean fever. Rheumatol Int. 2006;26(6):489-96.

14. Migita K, Nakamura T, Maeda Y, Miyashita T, Koga T, Tanaka M, et al. MEFV mutations in Japanese rheumatoid arthritis patients. Clin Exp Rheumatol. 2008;26(6):1091-4. 
15. Hershko AY, Ben-Chetrit E. The MEFV E148Q allele: a deleterious mutation or harmless variation? Clin Exp Rheumatol. 2006;24(5 Suppl 42):S51-2.

16. Marek-Yagel D, Bar-Joseph I, Pras E, Berkun Y. Is E148Q a benign polymorphism or a disease-causing mutation? J Rheumatol. 2009;36(10):2372.

17. Dember $L M$. Emerging treatment approaches for the systemic amyloidoses. Kidney Int. 2005;68(3):1377-90.

18. Duarte C, Gomes C, Correia AJ, Salgado M. Renal amyloidosis: an uncommon complication of juvenile idiopathic arthritis. Clin Rheumatol. 2006;25(4):548-9.

19. Saha A, Chopra Y, Theis JD, Vrana JA, Sethi S. AA amyloidosis associated with systemic-onset juvenile idiopathic arthritis. Am J Kidney Dis. 2013;62(4): 834-8.

20. Billiau AD, Cornillie F, Wouters C. Infliximab for systemic onset juvenile idiopathic arthritis: experience in 3 children. J Rheumatol. 2002;29(5):1111-4.

21. Kimura Y, Pinho P, Walco G, Higgins G, Hummell D, Szer I, et al. Etanercept treatment in patients with refractory systemic onset juvenile rheumatoid arthritis. J Rheumatol. 2005;32(5):935-42.

22. Russo RA, Katsicas MM. Clinical remission in patients with systemic juvenile idiopathic arthritis treated with anti-tumor necrosis factor agents. J Rheumatol. 2009;36(5):1078-82.

23. Russo RA, Katsicas MM, Zelazko M. Etanercept in systemic juvenile idiopathic arthritis. Clin Exp Rheumatol. 2002:20(5):723-6.

24. Fernandez-Nebro A, Olive A, Castro MC, Varela AH, Riera E, Irigoyen MV, et al. Long-term TNF-alpha blockade in patients with amyloid a amyloidosis complicating rheumatic diseases. Am J Med. 2010;123(5):454-61.

25. Gottenberg JE, Merle-Vincent F, Bentaberry F, Allanore Y, Berenbaum F, Fautrel $B$, et al. Anti-tumor necrosis factor alpha therapy in fifteen patients with AA amyloidosis secondary to inflammatory arthritides: a followup report of tolerability and efficacy. Arthritis Rheum. 2003;48(7):2019-24.

26. De Benedetti F, Brunner HI, Ruperto N, Kenwright A, Wright S, Calvo I, et al. Randomized trial of tocilizumab in systemic juvenile idiopathic arthritis. N Engl J Med. 2012;367(25):2385-95.

27. Yokota S, Imagawa T, Mori M, Miyamae T, Aihara Y, Takei S, et al. Efficacy and safety of tocilizumab in patients with systemic-onset juvenile idiopathic arthritis: a randomised, double-blind, placebo-controlled, withdrawal phase III trial. Lancet. 2008;371(9617):998-1006

28. Courties A, Grateau G, Philippe P. Flipo RM, Astudillo L, Aubry-Rozier B, et al. AA amyloidosis treated with tocilizumab: case series and updated literature review. Amyloid. 2015;22(2):84-92.

29. Okuda Y, Ohnishi M, Matoba K, Jouyama K, Yamada A, Sawada N, et al. Comparison of the clinical utility of tocilizumab and anti-TNF therapy in AA amyloidosis complicating rheumatic diseases. Mod Rheumatol. 2014;24(1): 137-43.

30. Okuda Y, Takasugi K. Successful use of a humanized anti-interleukin-6 receptor antibody, tocilizumab, to treat amyloid a amyloidosis complicating juvenile idiopathic arthritis. Arthritis Rheum. 2006;54(9):2997-3000.

31. Nowak B, Jeka S, Wiland P, Szechinski J. Rapid and complete resolution of ascites and hydrothorax due to nephrotic syndrome caused by renal amyloidosis in a patient with juvenile chronic arthritis treated with adalimumab. Joint Bone Spine. 2009:76(2):217-9.

32. Hakala M, Immonen K, Korpela M, Vasala M, Kauppi MJ. Good medium-term efficacy of tocilizumab in DMARD and anti-TNF-alpha therapy resistant reactive amyloidosis. Ann Rheum Dis. 2013;72(3):464-5.

\section{Submit your next manuscript to BioMed Central and we will help you at every step:}

- We accept pre-submission inquiries

- Our selector tool helps you to find the most relevant journal

- We provide round the clock customer support

- Convenient online submission

- Thorough peer review

- Inclusion in PubMed and all major indexing services

- Maximum visibility for your research

Submit your manuscript at www.biomedcentral.com/submit

C Biomed Central 